\section{UJMM

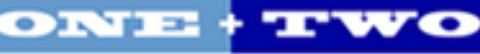

Volume 4 | 2011 Fall

\section{Undergraduate Journal of Mathematical}

Modeling: One + Two

2011

\title{
Length of a Hanging Cable
}

Eric Costello

University of South Florida

Advisors:

Arcadii Grinshpan, Mathematics and Statistics

Frank Smith, White Oak Technologies

Problem Suggested By: Frank Smith

Follow this and additional works at: https://digitalcommons.usf.edu/ujmm

Part of the Mathematics Commons

UJMM is an open access journal, free to authors and readers, and relies on your support:

Donate Now

\section{Recommended Citation}

Costello, Eric (2011) "Length of a Hanging Cable," Undergraduate Journal of Mathematical Modeling: One + Two: Vol. 4: Iss. 1, Article 4.

DOI: http://dx.doi.org/10.5038/2326-3652.4.1.4

Available at: https://digitalcommons.usf.edu/ujmm/vol4/iss1/4 


\title{
Length of a Hanging Cable
}

\begin{abstract}
The shape of a cable hanging under its own weight and uniform horizontal tension between two power poles is a catenary. The catenary is a curve which has an equation defined by a hyperbolic cosine function and a scaling factor. The scaling factor for power cables hanging under their own weight is equal to the horizontal tension on the cable divided by the weight of the cable. Both of these values are unknown for this problem. Newton's method was used to approximate the scaling factor and the arc length function to determine the length of the cable. A script was written using the Python programming language in order to quickly perform several iterations of Newton's method to get a good approximation for the scaling factor.
\end{abstract}

\section{Keywords}

Hanging Cable, Newton's Method, Catenary

\section{Creative Commons License}

(c) (i) (3)

This work is licensed under a Creative Commons Attribution-Noncommercial-Share Alike 4.0 License. 


\section{TABLE OF CONTENTS}

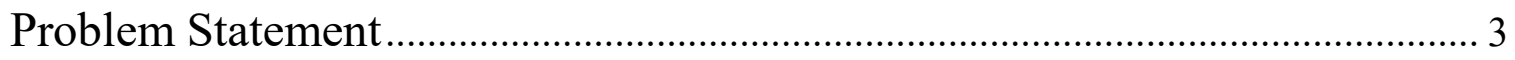

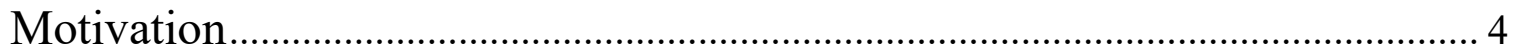

Mathematical Description and Solution Approach …………………................. 5

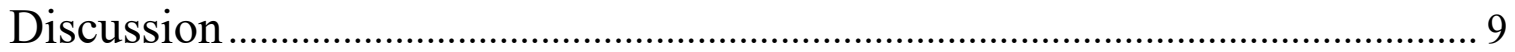

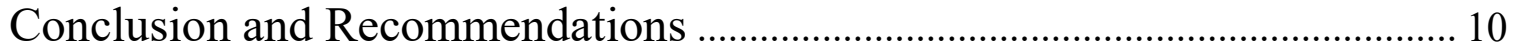

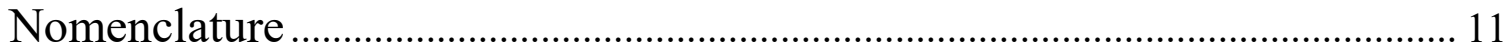

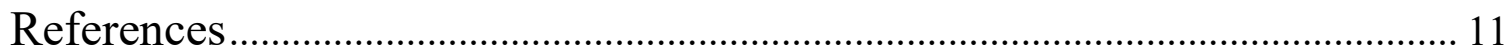

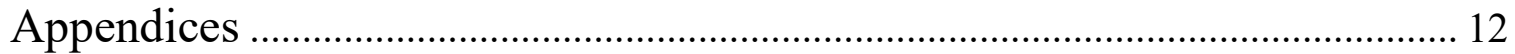




\section{PROBLEM STATEMENT}

A cable hangs between two $40 \mathrm{ft}$ power poles whose bases are on the same level, similar to the photograph below. The sag in the middle of the cable is $5 \mathrm{ft}$ and the towers are $100 \mathrm{ft}$. apart. Find the length of the cable in terms of the quotient $\frac{T_{0}}{w}$, where $\frac{T_{0}}{w}$ is the lowest point of the catenary on the y-axis. $T_{0}$ is the horizontal tension on the cable at its lowest point and $\mathrm{w}$ is the weight of the cable. Use Newton's method to approximate $\frac{T_{0}}{w}$ and find the length of the cable accurate to within $0.1 \mathrm{ft}$. Write a computer program to iterate with Newton's method and solve for the length of the cable.

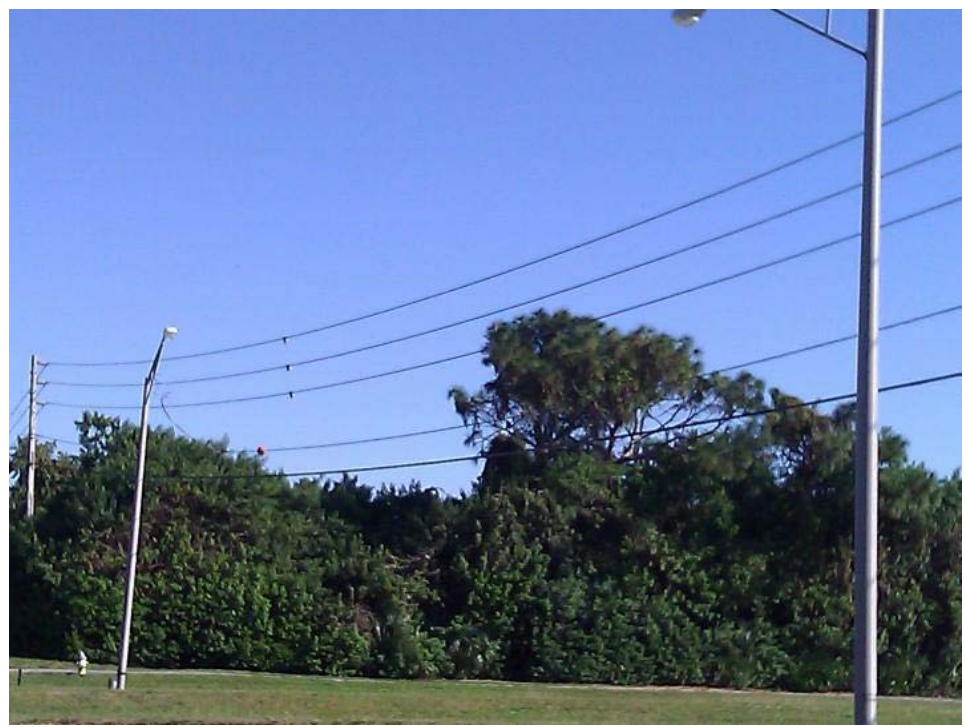

Figure 1: A cable hanging between to power poles. 


\section{MOTIVATION}

Transmission of electrical power is a necessary component of life in modern society. The North American Electric Reliability Corporation (NAERC) stated in their 2009 assessment that due to the increase in power generating plants over the next ten years, there is a need to begin increasing the transmission capability of our energy infrastructure (WWW.NERC.COM). These transmission lines must be maintained and replaced by the power producers. In order to minimize the cost of replacement, the power producers must understand how these power lines behave when hanging on transmission poles, so that they can design a system to ensure the lines last long while using the shortest length of cable possible.

This project is designed to determine the length of a cable spanning two power poles when the tension and cable weight are not known. In applications such as this, the value $\frac{T_{0}}{w}$ is commonly referred to as the catenary constant. This value varies based on environmental conditions. However, it is typically in the range of several thousand feet for most transmission line catenaries (Grigsby). Based on this information, it is required to suggest an initial value for $\frac{T_{0}}{w}$ and then iterate with Newton's Method until an approximation of the value approaches a value within the margin of the error. A sufficient number of iterations performed by hand to get a decent approximation is a very tedious and time consuming process. The calculation process can be accomplished fast, with greater accuracy using a computer program. The goal of this project is to define the appropriate mathematical relations and find the cable length with the aid of a computer program. 


\section{MATHEMATICAL DESCRIPTION AND SOLUTION APPROACH}

The equations needed to solve this problem were taken from Essential Calculus: Early

Transcendental (Stewart):

$$
\begin{array}{ll}
\text { Arc length formula: } & l=\int_{a}^{b} \sqrt{1+\left[f^{\prime}(x)\right]^{2}} d x \\
\text { Catenary equation: } & f(x)=a \cosh \left(\frac{x}{a}\right)
\end{array}
$$

$$
\text { Newton's iteration formula: } \quad x_{n+1}=x_{n}-\frac{g(x)}{g^{\prime}(x)}
$$

The formula for arc length (Eq. 1) can be used to determine the length of the cable in terms of $\frac{T_{0}}{w}$.

The shape of the arc is given by the catenary function (2). The derivative of (2) is,

$$
f^{\prime}(x)=\sinh \left(\frac{x}{a}\right)
$$

In order to simplify the following equations, the variable $a=\frac{T_{0}}{w}$ can be substituted into Eq. 1 .

The integral we need to solve in order to determine the cable length is,

$$
l=\int_{-50}^{50}\left\lfloor\sqrt{1+\left(\frac{d}{d x} a \cosh \left(\frac{x}{a}\right)\right)^{2}}\right\rfloor d x
$$

Substituting $f^{\prime}(x)$ into Eq. (5),

$$
l=\int_{-50}^{50} \sqrt{1+\left[\sinh \left(\frac{x}{a}\right)\right]^{2}} d x
$$

Substituting the identity $1+\sinh ^{2} x=\cosh ^{2} x$ to the Eq. (6)

$$
l=\int_{-50}^{50} \cosh \left(\frac{x}{a}\right) d x
$$


Since $\cosh (x)$ is an even function, by symmetry, this integral is equivalent to,

$$
l=2 \int_{0}^{50} \cosh \left(\frac{x}{a}\right) d x=2\left[a \sinh \left(\frac{x}{a}\right)\right]_{0}^{50}=2 a \sinh \left(\frac{50}{a}\right)
$$

The final cable length formula $l$ in terms of $\frac{T_{0}}{w}$ is,

$$
l=2 \frac{T_{0}}{w} \sinh \left(50 \frac{w}{T_{0}}\right)
$$

Since the horizontal tension $\left(T_{0}\right)$ or the weight of the cable $(w)$ was not given, Newton's Method is used to approximate the value for $\frac{T_{0}}{w}$. The approximated value then applies in Eq. 8, to find the length of the cable accurate within $0.1 \mathrm{ft}$. For this purpose, function $g(a)$ is defined such that the zero of $g(a)$ gives the value for $\frac{T_{0}}{w}$. Then, Newton's Method is used to find where the value of $g(a)$ function reaches zero.

The formula for Newton's Method in terms of $a\left(a=\frac{T_{0}}{w}\right)$ is:

$$
a_{n+1}=a_{n}-\frac{g(a)}{g^{\prime}(a)}
$$

According to the problem, the power poles are $40 \mathrm{ft}$. high and are situated $100 \mathrm{ft}$. apart. The sag in the middle is $5 \mathrm{ft}$. and the midpoint is $\frac{T_{0}}{w} \mathrm{ft}$ above the horizontal axis (x-axis). Using this information, the following equations can be generated.

Since the cable sags $5 \mathrm{ft}$. from its highest points, which are located at $f(-50)$ and $f(50)$ :

$$
f(0)=f(50)-5
$$

Substituting $x$ values in to the catenary equation (Eq. 2): 


$$
\begin{gathered}
f(50)=a \cosh \left(\frac{50}{a}\right) \\
f(0)=a \cosh \left(\frac{0}{a}\right)=a
\end{gathered}
$$

Substituting Eq. 11 and 12 into Eq.10,

$$
\begin{gathered}
a=a \cosh \left(\frac{50}{a}\right)-5 \\
0=a+5-a \cosh \left(\frac{50}{a}\right)
\end{gathered}
$$

The right hand side of the Eq. (14) is equal to zero which satisfies the conditions in the problem.

$$
\text { Thus, define } g(a) \text { as, } \quad g(a)=a+5-a \cosh \left(\frac{50}{a}\right)
$$

The Eq. 15 can use to iterate with Newton's Method. The derivative of $g(a)$,

$$
g^{\prime}(a)=1-\cosh \left(\frac{50}{a}\right)+50 a \sinh \left(\frac{50}{a}\right)
$$

Thus, the standard iteration formula can be written as:

$$
a_{n+1}=a_{n}-\frac{a+5-a \cosh \left(\frac{50}{a}\right)}{1-\cosh \left(\frac{50}{a}\right)+50 a \sinh \left(\frac{50}{a}\right)}
$$

Since the tension and the weight were unknown, a Python script (Appendix A) was written to iterate Newton's Method. The calculation began using Newton's Method with the initial value of $a_{n}=20$. As seen in Fig. 1, $g(a)$ is a smooth function. The zero of this function should converge to a specific point due to this reason. The function $g(a)$ was plotted using Google's graphing calculator in order to determine an approximate zero of this function, and thus determine when to stop iterating. We could also anticipate that our value should be around $250 \mathrm{ft}$. 


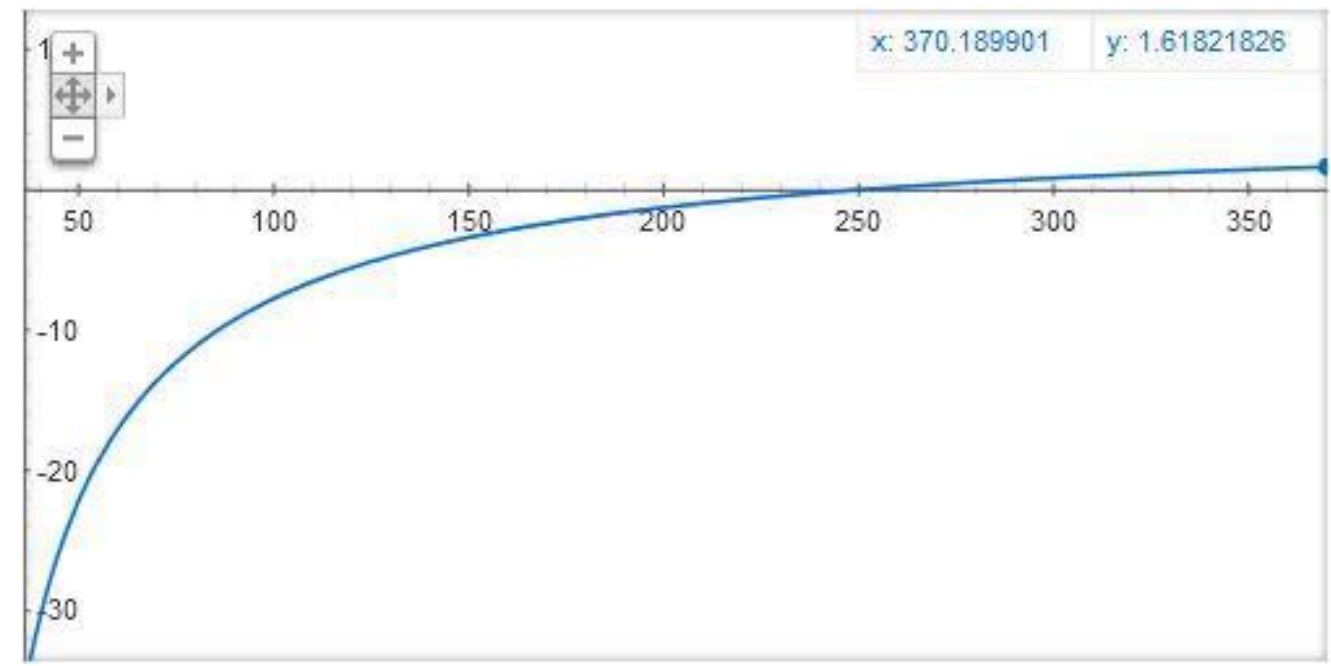

Figure 1: Graph of variation of function $g(a)$ with $\mathrm{x}$.

\begin{tabular}{|c|ccc|}
\hline $\boldsymbol{g}(\boldsymbol{a})$ & $\boldsymbol{g}^{\prime}(\boldsymbol{a})$ & $\boldsymbol{a}_{\boldsymbol{n}}$ & $\boldsymbol{a}_{\boldsymbol{n}+\mathbf{1}}$ \\
\hline-50.244 & 4.492 & 20.000 & 31.186 \\
\hline-44.438 & 2.237 & 31.186 & 51.0518 \\
\hline-21.506 & 0.601 & 51.052 & 86.841 \\
\hline-9.796 & 0.180 & 86.841 & 141.342 \\
\hline-3.936 & 0.065 & 141.342 & 202.332 \\
\hline-1.209 & 0.031 & 202.332 & 241.345 \\
\hline-0.198 & 0.022 & 241.345 & 250.467 \\
\hline-0.007 & 0.020 & 250.467 & 250.828 \\
\hline$-1.06 \mathrm{E}-05$ & 0.020 & 250.828 & 250.829 \\
\hline
\end{tabular}

Table 1: Values for Newton's method

The iteration values converge in to approximate value of $\frac{T_{0}}{w}=250.8289318$ after nine iterations.

We used this value in Eq.8 to determine that the length of the cable is equal to $100.67 \mathrm{ft}$. 


\section{DISCUSSION}

The cable length value $(100.67 \mathrm{ft})$ obtained from this method quite surprised us as it is very close to the distance between poles. In order to check the accuracy of our results another method was adopted. The Riemann sum was calculated for two intervals and four intervals based on right triangles formed by the information given in the problem. In order to calculate the Riemann sum, endpoints for two and four intervals of equal length were found using Eq. 2. For each interval, a right triangle was constructed by drawing a vertical line through the lower endpoint, a horizontal line through the higher endpoint and a line connecting the points as shown in Fig. 2 and Fig. 3. The length of the hypotenuse is a linear approximation to the arc length of the section. For two intervals, these triangles have the sides $\Delta y=5 \mathrm{ft}$ and $\Delta x=50 \mathrm{ft}$. hypotenuse length, 50.24937811 was calculated using the Pythagorean theorem. The total length calculated as $100.499 \mathrm{ft}$. by symmetry. The same procedure repeated for four intervals and the results obtained as $100.622 \mathrm{ft}$. The detailed calculations for these sums are included in Appendix B. This quick approximation verifies the value calculated using Newton's method is accurate.

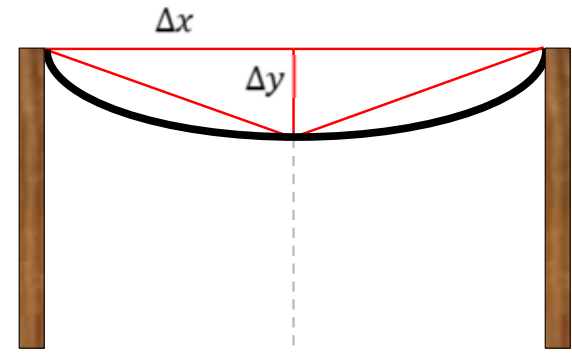

Figure 2: Approximating Triangles for Riemann Sum, 2 intervals (Not to Scale)

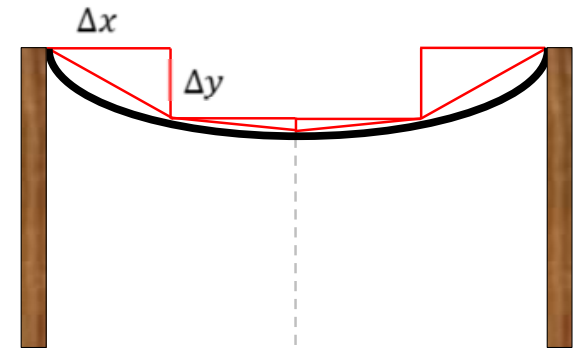

Figure 3: Approximating Triangles for Riemann Sum, 4 intervals (Not to Scale) 


\section{CONCLUSION AND RECOMMENDATIONS}

At first, this problem seems to be relatively straight-forward. We made the assumption that the $\mathrm{x}$ axis lies on the ground; therefore, determining a value for $\frac{T_{0}}{w}$ would simply involve subtracting the amount of sag from the maximum height. While researching this problem, it was discovered that this is not the case, and the $\mathrm{x}$-axis actually lies underground. This makes the problem much more difficult since the value $\frac{T_{0}}{w}$ cannot be algebraically separated from the hyperbolic cosine in the equation $f(x)=\frac{T_{0}}{w} \cosh \left(\frac{w}{T_{0}} x\right)$.

By understanding the relationship of tension vs. cable weight to the length of the cable needed, power companies can design a viable transmission system while minimizing the cost involved in maintenance while reducing wasted cable. For example, if the amount of tension a cable can withstand is known, the engineer can use these same functions to optimize the distance between poles and length of the cable in order to design the most cost effective, yet reliable system for the power grid. Real world engineering problems such as this one would also require that the engineer consider environmental factors such as wind loads, changes in tension due to poles shifting and ice build-up. However; the resulting optimization problem is beyond the scope of this project.

Newton's method is a powerful tool to approximate zeros of a function; however it is tedious to do by hand. By using a small script to iterate through it, and setting the correct parameters to stop iterating, it can be accomplished quickly and easily. 


\section{NOMENCLATURE}

\begin{tabular}{|clc|}
\hline Symbol & Description & Units \\
\hline $\boldsymbol{T}_{\mathbf{0}}$ & Horizontal Tension & N \\
\hline $\boldsymbol{w}$ & Weight & lbs \\
\hline
\end{tabular}

\section{REFERENCES}

Grigsby, Leonard L. The Electric Power Engineering Handbook. Taylor and Francis, 2001.

J. Douglas Faires, Barbara Trader Faires. Calculus. McGraw-Hill, 1992.

Stewart, James. Essential Calculus: Early Transcendentals . Brooks/Cole , 2010.

www.nerc.com. 2010. $23112011<$ http://www.nerc.com/files/2010\%20LTRA.pdf>. 


\section{APPENDICES}

\section{APPENDIX A - PYTHON CODE}

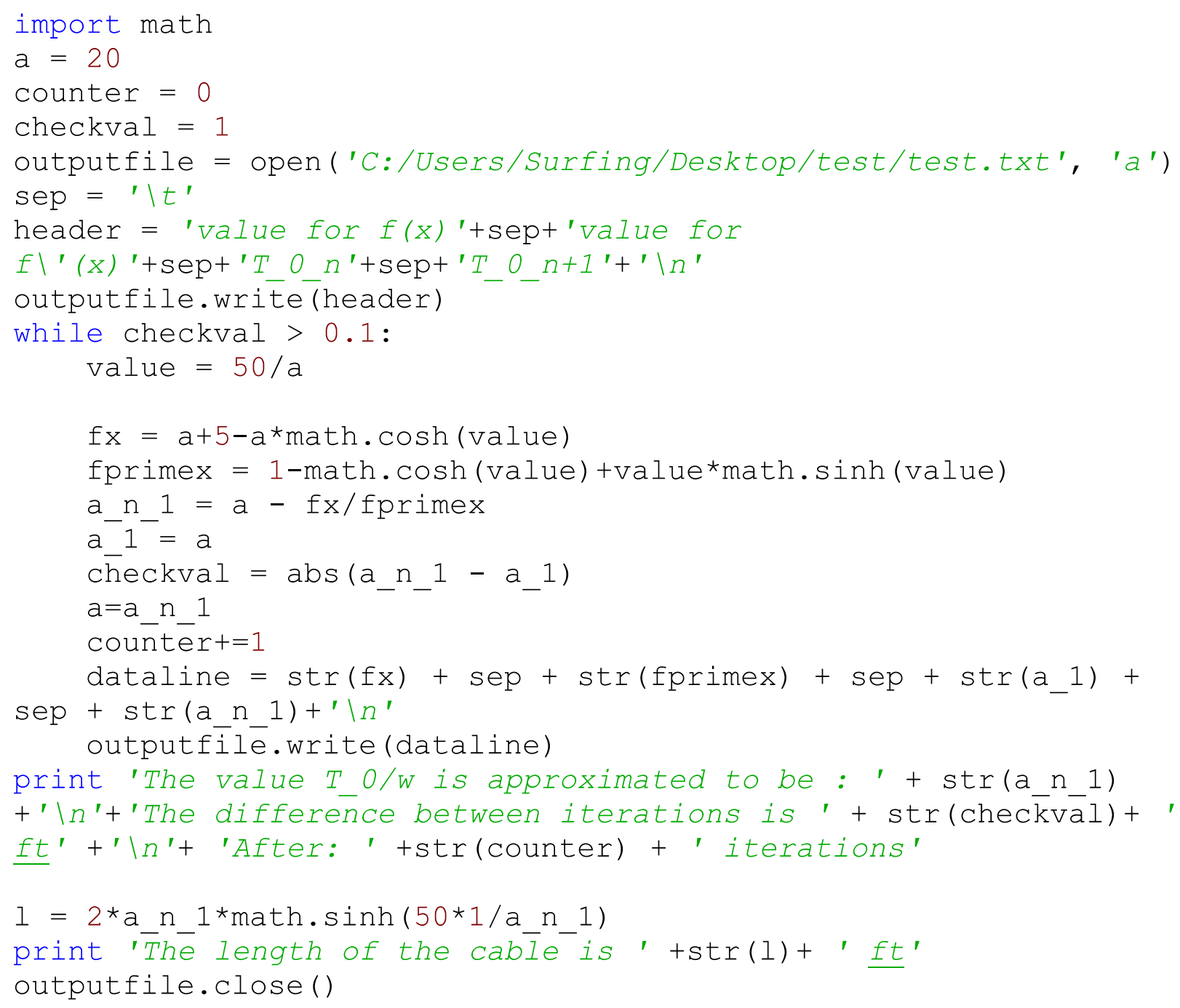




\section{APPENDIX B - RIEMANN SUM CALCULATIONS}

$$
\begin{gathered}
f(0)=250.828 \\
f(25)=250.828 \cosh \left(\frac{25}{250.828}\right)=252.075 \\
f(50)=250.828 \cosh \left(\frac{50}{250.828}\right)=255.828 \\
\sum_{i=1}^{2} \sqrt{\Delta x_{i}{ }^{2}+\left[\Delta f\left(x_{i}\right)\right]^{2}}=2 \sqrt{\Delta x^{2}+[f(50)-f(0)]^{2}}=2 \sqrt{2525}=100.498 f t \\
\sum_{i=1}^{4} \sqrt{\Delta x_{i}{ }^{2}+\left[\Delta f\left(x_{i}\right)\right]^{2}}=2\left[\sqrt{\Delta x^{2}+[f(25)-f(0)]^{2}}+\sqrt{\Delta x^{2}+[f(50)-f(25)]^{2}}\right. \\
=2(\sqrt{626.555}+\sqrt{639.085})=100.622 f t
\end{gathered}
$$

